\title{
STUDY ON THE INTERACTION MECHANISM OF FLOW AND SEDIMENT IN AN OPEN CHANNEL CONTAINING VEGETATION
}

\author{
QU, G. ${ }^{*}-$ ZHAO, Z. C. - YAO, S. M. - ZHU, Y. H. - ChEN, D. \\ Changjiang River Scientific Research Institute, Wuhan, Hubei 430010, China \\ ${ }^{*}$ Corresponding author \\ e-mail: qugeng0516@163.com
}

(Received $19^{\text {th }}$ Apr 2019; accepted 11 $1^{\text {th }}$ Jul 2019)

\begin{abstract}
The understanding of the effect of vegetation on the flow and sediment transport is extremely important for modern river management and water ecological restoration. In this study, the laboratory experiments are carried out to study the 3D flow velocity, turbulence and sediment transport affected by 3 types of flexible vegetation. The results show that the submerged and emergent vegetation generates an obvious flow resistance and significantly alters the vertical distributions of the flow velocity, especially in the vegetated and downstream regions. Due to the influence of vegetation, the flow in downstream generates many vortices with different sizes. Compared with the submerged vegetation, the emergent vegetation has a more obvious influence on the vortex flow. Vegetation also results in a nearly constant fine suspended sediment concentration in the water column for all cases, while the large flow resistance has a stronger filtration effect on the coarse sediment, which leads the coarse sediment to be deposited and the total sediment along the vegetated channel to be gradually refined. The experiment also analyzes the influence of the transverse flow on the suspended sediment transport and the interaction mechanism of flow and sediment.
\end{abstract}

Keywords: vegetation, flow velocity, turbulence, sediment transport, vortex flow, diffusion coefficient

\section{Introduction}

Vegetation can be found in abundance in the shore and shallow water region of rivers and plays an important role in influencing the turbulent flow field in a river channel. At present, as people pay more and more attention to the ecological protection of rivers, the research on the problems of flow and sediment transport in vegetated rivers has become one of the hottest topics in river dynamics. For a river, the vegetation tends to increase the flow resistance during the flood periods, increase the water level and thus reduce its overcurrent capacity. Besides, with the growth of vegetation and the increasing resistance, it will change the local flow and the distribution of sediment transport. Therefore, it is important to carry out research on the characteristics of flow and sediment transport in vegetated rivers. The open channel flow movement is usually a boundary layer turbulence. Under certain channel profile conditions (e.g. straight and shallow channel), it can be considered as a two-dimensional flow (Wang and Li, 2002). While affected by the physical and distribution characteristics of vegetation, the flow in 
the vegetated open channel has strong properties of three-dimensional motion. The main differences in the characteristics of the two flow motions are the distribution of flow characteristic parameters, such as flow velocity, turbulence intensity, Reynolds stress, etc. (Stephan and Gutknecht, 2002; Carollo, 2002; Tang et al., 2007. The distribution of the vegetated flow has two or three divisions of the zone in the vertical direction, and the whole distribution in vertical direction does not follow the logarithmic distribution any more. Ikeda and Kanazawa (1996) conducted laboratory experiments on the flow characteristics of flexible vegetation and found that eddies and flow velocity changed significantly near the vegetation. Nezu and Onitsuka (2001) studied the turbulent flow structure in the vegetated open channel and found that the horizontal vortex near free surface was caused by the increase of Froude number and vegetation density. In terms of the maximum turbulence intensity of the flow Ikeda and Kanazawa (1996) found that the maximum turbulence intensity appeared at the top of vegetation, which was consistent with some experimental and numerical simulations. However, some researchers demonstrated that the maximum turbulence intensity of the vegetated flow was generated at the junction of the vegetation's top and non-vegetation zone ( $\mathrm{Su}$ and $\mathrm{Li}, 2002$ ). Wilson et al. (2003) proposed that the position of maximum turbulence tends to move toward the free surface with the increase of submergence, for the flexible vegetation with leaves. Sediment transported in the vegetated open channel has also drawn the attention of many researchers. Shi and Cao (2000) found that the presence of vegetation led to a decrease of the coefficient of turbulent diffusion and a significant increase of gravitation. In his opinion, the vegetation can not only block the bed-load transport effectively but also intercept the suspended sediment movement. However, experimental studies by Elliott (2000) on the effects of sedimentation rate by vegetation show that compared with non-vegetation, the presence of vegetation reduced sedimentation rate and made sediment more easily suspended. Lu (2008) investigated the suspended sediment transport in the rigid vegetated open channel and analyzed the influence of vegetation factors on the distribution of turbulence and sediment concentration. Jordanova and James (2003) established an empirical formula for the rate of bed-load transport in non-submerged rigid vegetated open channel by using the analogy of the equation of bed-load transport rate in non-vegetated rivers and his experimental data. Li and Shen (1973) found that compared with the parallel arrangement of vegetation in the open channel, the plum blossom staggered arrangement could obstruct the flow more efficiently and the amount of sediment transport was also relatively small. Therefore, when considering flood control and soil conservation of rivers, the vegetation arrangement in the channel should be reasonably designed.

There is plenty of research on the characteristics of flow and sediment transport in the vegetated open channels at present. Due to the complexity of vegetation morphology and the difficulties of using numerical simulation methods, there are relatively more studies using the experimental flumes. However, there are some problems that still need to be further studied: (1) There are many kinds of aquatic vegetation in river ecosystem, but at present, most of the research mainly focuses on specific types of vegetation, and 
there are few research results on both submerged and emergent vegetation. Therefore, systematic research needs to be strengthened. (2) The research on the turbulent structure of three-dimensional flow in the vegetated open channel has been very thorough, but it is limited to the problems of sediment sample collections and measurement equipment. There is little research on the characteristics of flow and sediment transport within the upstream and downstream region in the partly vegetated open channel. In this paper, three representative submerged and emergent artificial flexible vegetation is used to carry out experimental studies on the characteristics of flow and sediment transport in a partly vegetated open channel. The three-dimensional distribution of flow movement, suspended sediment transport in the partly vegetated open channel is obtained. Based on these, the mechanism of interaction between flow and sediment transport in the partly vegetated open channel is further discussed. These research results aim at further enriching the understanding of the regularity of flow and sediment transport in the vegetated channel and lay a foundation for further research.

\section{Material and Methods}

\section{Experimental equipment}

All the experiments are conducted in the Hydraulics Laboratory in Changjiang River Scientific Research Institute, Wuhan, China. The experimental apparatus is mainly composed of three pumps, an inlet section to generate fully developed turbulent flow, a test section with a rectangle flume to operate interaction between flow and vegetation, an automatic control system installed at the inlet of the rectangle flume to control the amount of sediment. The re-circulating flume is $0.5 \mathrm{~m}$ wide, $0.5 \mathrm{~m}$ high and $28 \mathrm{~m}$ long, with glass sidewalls and bottom (Figure 1). The range of the flume slope is $0 \%$-5\%o. Thin plastic board is laid on the bottom of the flume, on which the simulated vegetation is planted. In order to decrease the inlet turbulence and maintain the uniform flow, a wave dissipation board is set at the inlet of the flume. An automatic water level control system located at the outlet of the flume is used to accurately control the water level. Preliminary experiments are carried out to ensure there is no flow turbulence effect due to the thin plastic board within the measurement region. It is long enough far from the inlet to ensure that the flow through the vegetation is smooth and the measurement data is accurate. A three dimensional Macro-Acoustic Doppler Velocimetry (ADV) (SonTek, San Diego, CA, USA) is used to measure the flow velocity and turbulence at a frequency of $25 \mathrm{~Hz}$ with 30 second (s) sampling time. From the initial control experimental run, it is revealed that the sampling frequency is enough to obtain accurate velocity and turbulence characteristics ( $\mathrm{Li}$ et al., 2015). Thus, 750 data measurements are collected at every location and with post-processing software (WinADV), and an average velocity value is obtained. Measurements at $5 \mathrm{~cm}$ below the free surface can not be taken due to ADV limitations. For more information about the application of ADV on flow measurement in a flume, refer to (Qu, 2014). The measuring equipment used in the experiment include electromagnetic flowmeter, the ADV three-dimensional 
flowmeter, the automatic water level meter, Sediment sampler (The instrument uses the siphon principle for sediment sampling), Malvern Mastersizer 2000 particle size analyzer (The instrument uses laser diffraction technology to measure particle size of sediment) and the electronic balance.

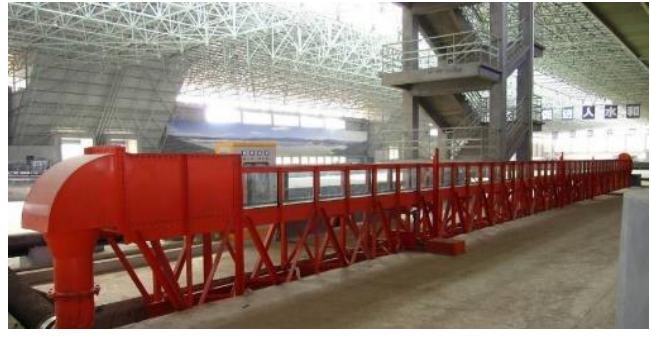

(a) Flume

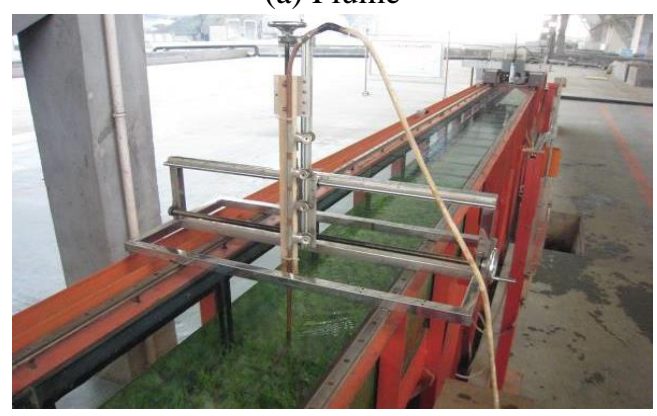

(c) Sediment Collector

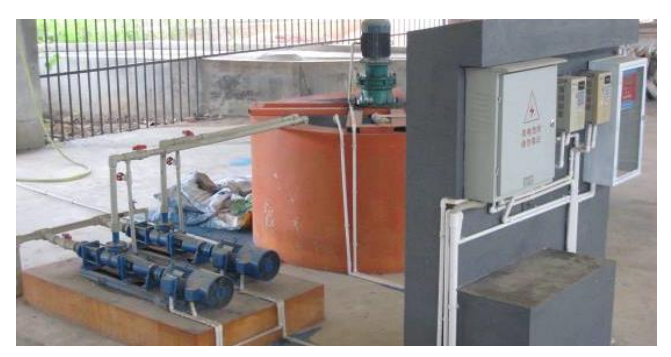

(b) Sediment Suppler

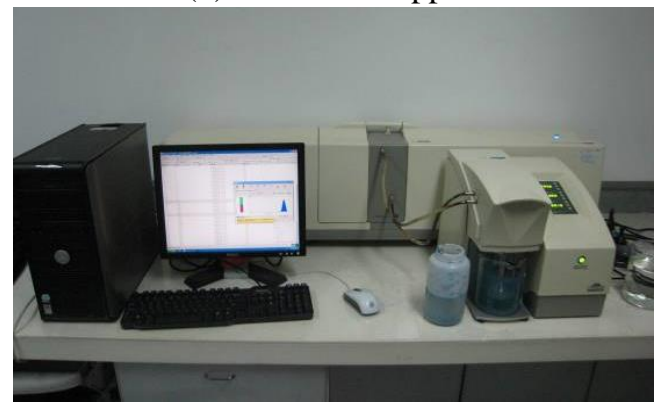

(d) Particle Sizer Analysis System

Figure 1. Experimental equipment and instruments

\section{Experimental program}

The experiment investigates the affection of the effects on the flow and sediment transport in the open channel, based on the acquired laboratory data conducted using three artificial flexible vegetation (two kinds of submerged flexible vegetation, and one kind of emergent flexible vegetation). The three kinds of artificial vegetation use the same flexible plastic material (the material proportion is $1.35 \mathrm{~g} / \mathrm{cm}^{3}$, the elastic modulus is $0.03 \times 10^{5} \mathrm{Mpa}$ ). This kind of plastic vegetation has foliage and flexibility characteristics like true plants, which are representative of the vegetation in the riparian zone of rivers, and can simulate nature vegetation drag force in open channel (Figure 2, Figure 3). The three kinds of vegetation are used in the experiments with the same arrangement, and ten measured sections are arranged in the partly vegetated open channel (Figure 4). The flow and sediment of experimental conditions are as follows: The inlet discharge of the flume is $30 \mathrm{~L} / \mathrm{s}$. The outlet water level of the flume is $35 \mathrm{~cm}$, and the amount of sediment at the inlet of the flume is $5.0 \mathrm{~g} / \mathrm{s}$. The sediment using in the experiment is a kind of plastic synthetic sand, its wet and dry density is $1.38 \mathrm{t} / \mathrm{m}^{3}, 0.67$ $\mathrm{t} / \mathrm{m}^{3}$, respectively (Sun and Wei, 2005). Figure $5 a$ shows the grading curves of the artificial sediment used for all cases and they follow a similar composition with a median particle size $\left(\mathrm{D}_{50}\right)$ of $0.18 \mathrm{~mm}$. The maximum slope of the flume using in this experiment is 5/1000. All the experiments are controlled according to the standard of 
uniform flow (the slopes of flume and water surface are the same). In order to obtain the approximately uniform flow if the slope of the water surface in the vegetated area is always larger than the maximum slope of the flume, then the flume is adjusted according to the maximum slope of 5/1000. The experimental results show that the slope of the water surface in upstream and downstream of the vegetated region is less than that in the vegetated region (Figure $5 b$ ), so the slope of the water surface in upstream and downstream region is less than the slope of the flume. The flow in the upstream and downstream region is the decelerating flow (Yang and Lee, 2007).

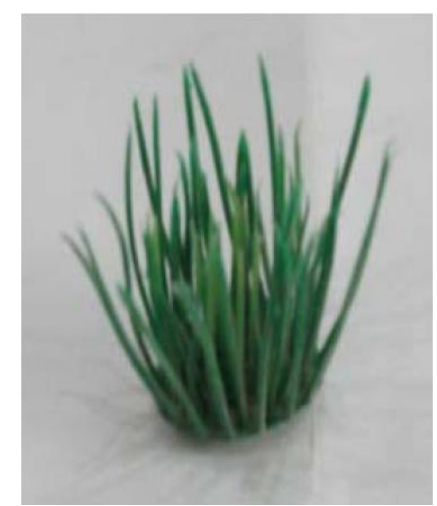

Vegetation P1

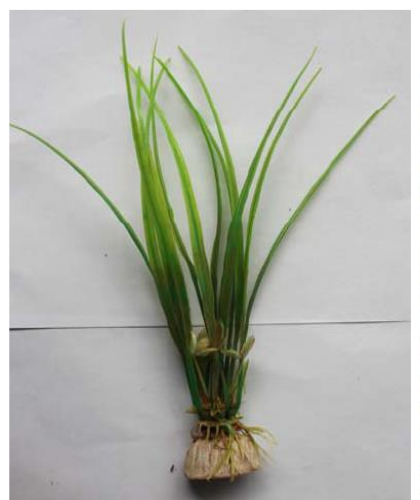

Vegetation P2

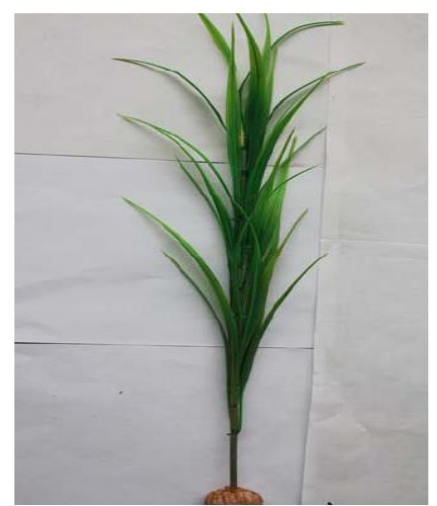

Vegetation P3

Figure 2. Image of artificial different vegetation
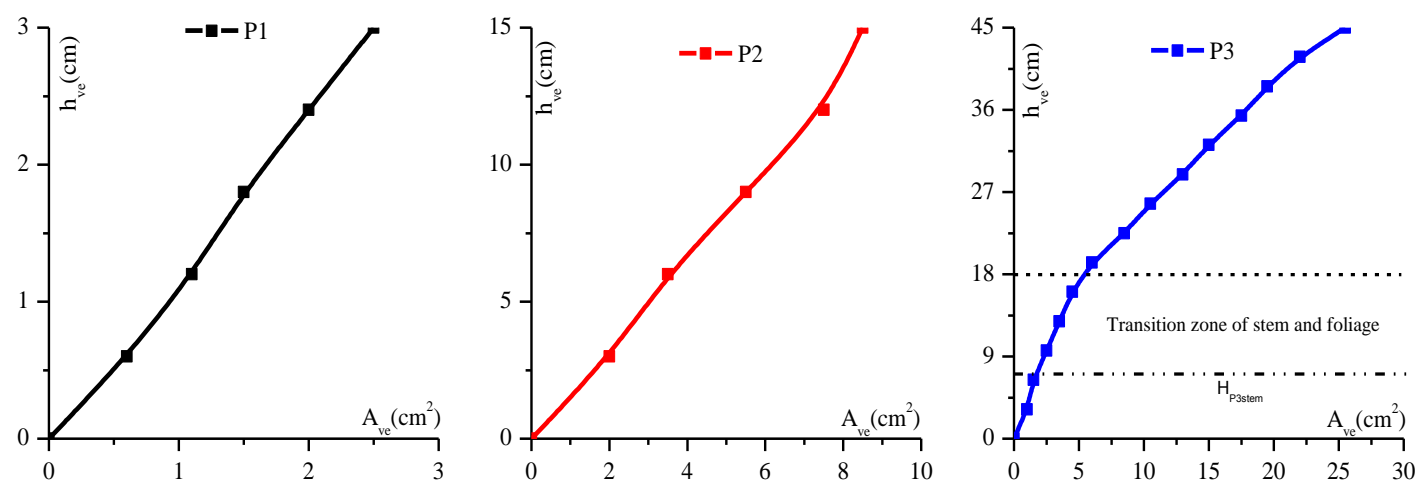

Figure 3. Profile of the variation of the vegetation cumulative area with respect to the height of vegetation

There are two points to note in this paper. (1) The flow pattern is different along the stream-wise in the partly vegetated open channel, however, the main research is on the effect of the flow and sediment transport in the partly vegetated channel. (2) The preliminary experiment shows that the sediment transport is basically balanced (the concentration of sediment at inlet and outlet of the open channel is basically the same) in the non-vegetated open channel under the experimental condition of Table 1 (Case 1). In order to facilitate the comparison with the flow and sediment transport in the open 
channel under different types of vegetation, the flow patterns in the upstream and downstream regions are approximately regarded as a uniform flow.

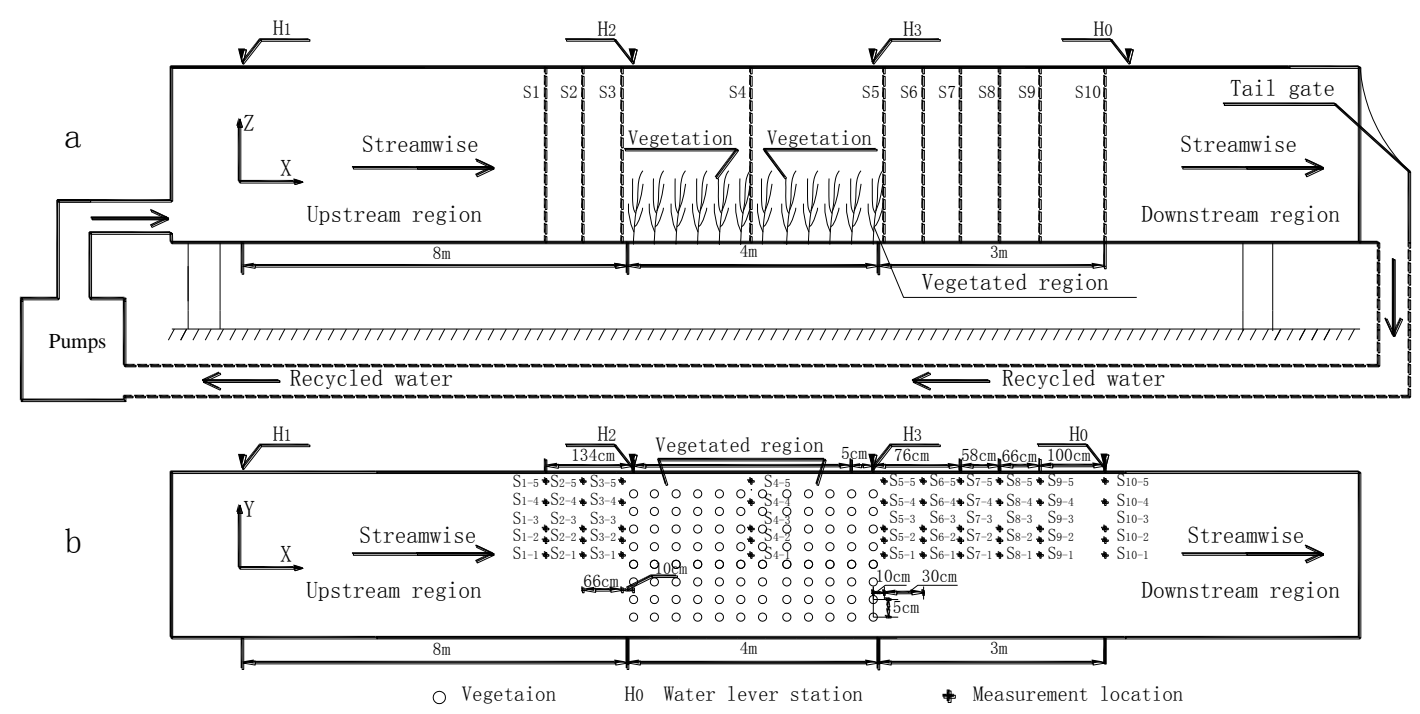

Figure 4. Schematic diagram of the experimental flume: (a) Side view; (b) Top view (not to scale)

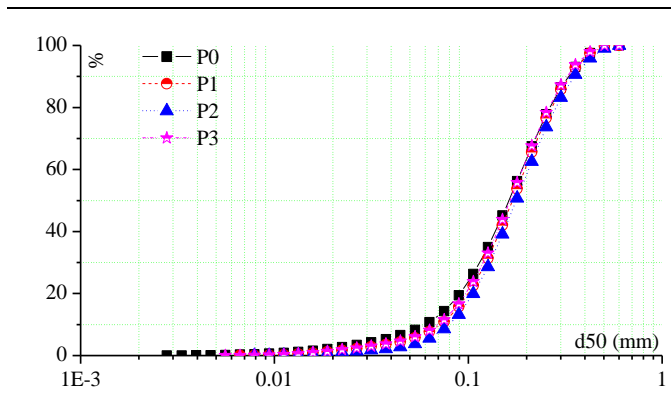

(a)

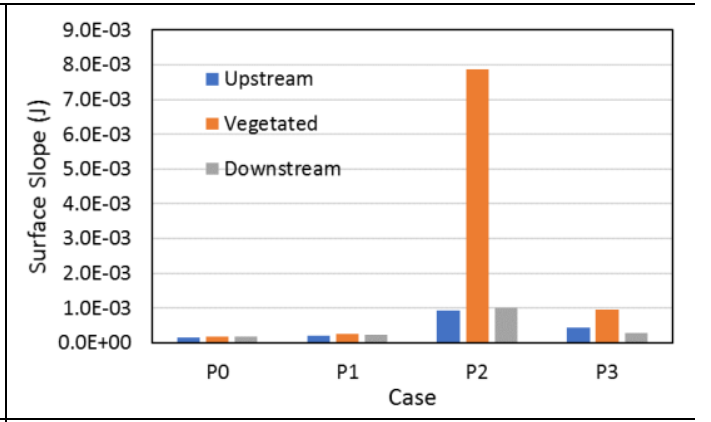

(b)

Figure 5. The grading curves of sediment used and slope of water surface in different regions under different conditions of vegetation

Table 1. The conditions of flow and sediment

\begin{tabular}{c|c|c|c|c|c|c|c|c}
\hline Case & Type & $\begin{array}{c}\mathbf{Q} \\
(\mathbf{L} / \mathbf{s})\end{array}$ & $\begin{array}{c}\mathbf{H o} \\
(\mathbf{c m})\end{array}$ & $\begin{array}{c}\mathbf{S a} \\
(\mathbf{g} / \mathbf{s})\end{array}$ & $\begin{array}{c}\mathbf{U a} \\
(\mathbf{c m} / \mathbf{s})\end{array}$ & $\begin{array}{c}\mathbf{J} \\
(\mathbf{1} / \mathbf{1 0 0 0})\end{array}$ & $\begin{array}{c}\mathbf{S}_{\mathbf{L}} \\
(\mathbf{1} / \mathbf{1 0 0 0})\end{array}$ & Remark \\
\hline 1 & P0 & 30 & 35 & 5.0 & 17.15 & 0.18 & 0.18 & Uniform flow \\
2 & P1 & 30 & 35 & 5.0 & 17.16 & 0.30 & 0.30 & Uniform flow \\
3 & P2 & 30 & 35 & 5.0 & 17.23 & 0.97 & 0.97 & Uniform flow \\
4 & P3 & 30 & 35 & 5.0 & 17.31 & 8.58 & 5.00 & $\begin{array}{c}\text { Approximately } \\
\text { uniform flow }\end{array}$ \\
\hline
\end{tabular}




\section{Results}

\section{Flow velocity characteristic}

\section{Longitudinal flow velocity}

(1) Flow velocity along stream-wise

To highlight the effect of artificial vegetation on the flow characteristics along the stream-wise, the longitudinal velocity data has been processed and plotted at different positions in the open channel. Figure 6 represents the vertical distribution of the uvelocity component normalized by $\mathrm{Ua}$ at different longitudinal positions in the midline of the open channel. For P0, the vertical distribution of longitudinal flow velocity from S1 to S10 cross-sections basically complies with the logarithmic distribution.

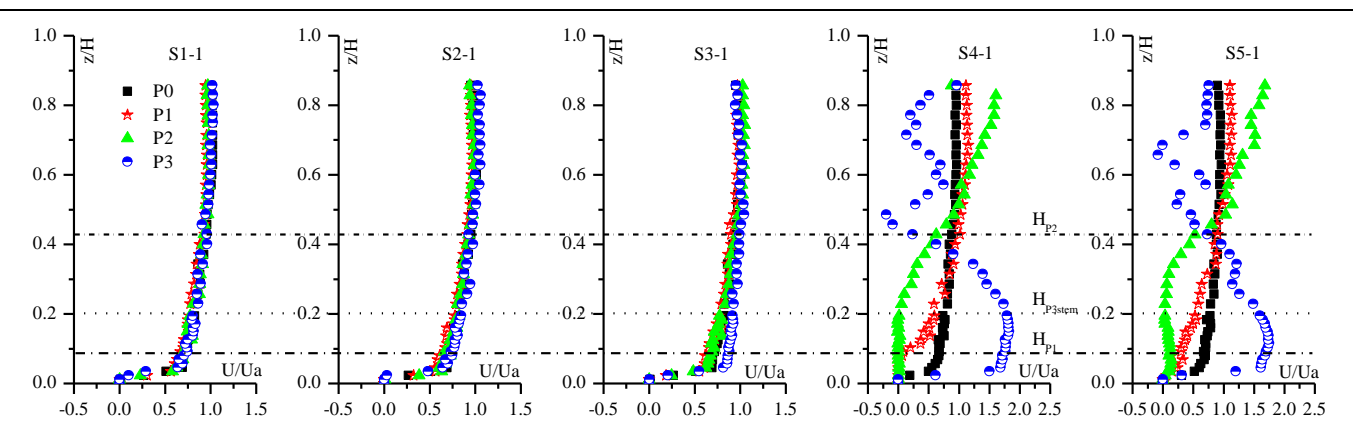

(a)

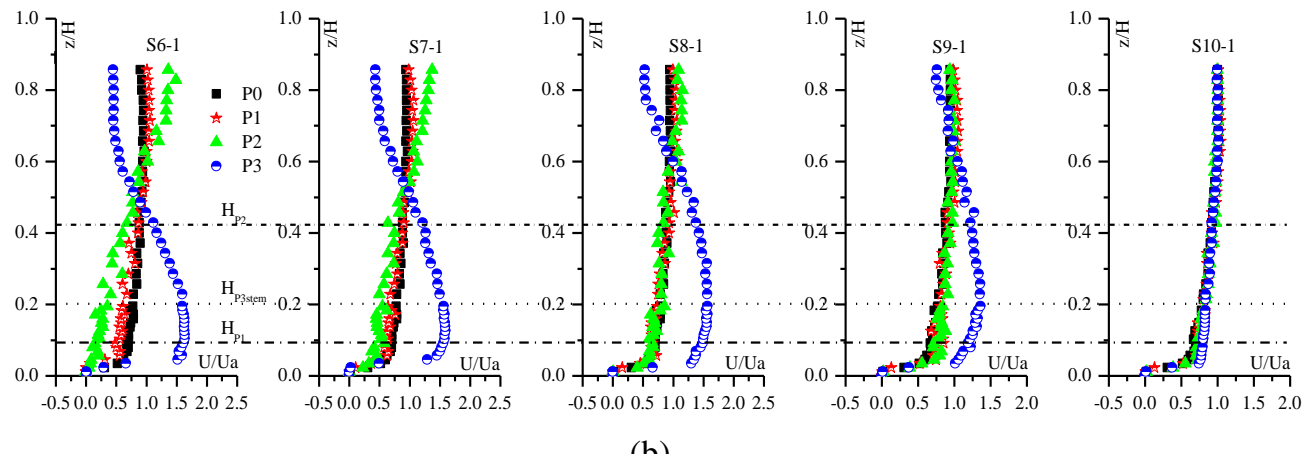

(b)

Figure 6. The vertical distribution of the normalized velocity u/ua changed along the streamwise in the vegetated open channel (a) from S1-1 to S5-1, (b) from S6-1 to S10-1

For $\mathrm{P} 1$, in upstream region (S1-1 to S3-1), the vertical distribution of longitudinal flow velocity is basically similar to that of $\mathrm{P} 0$; in the vegetation region (S4-1), the flow velocity in the zone below the height of $0.2 \mathrm{z} / \mathrm{H}$ is smaller than that of $\mathrm{P} 0$, however, the flow velocity in the zone above the height of $0.2 \mathrm{z} / \mathrm{H}$ is larger than that of P0. The vertical distribution of flow velocity in longitudinal direction basically satisfies the logarithmic distribution from the top of $\mathrm{P} 1$ to the free surface (above $0.1 \mathrm{z} / \mathrm{H}$ ). In the downstream region (at S5-1), the flow velocity in the zone below the height of $0.3 \mathrm{z} / \mathrm{H}$ reduces obviously. Since then, the more toward the downstream of the open channel, the flow velocity gradually approaches to that of $\mathrm{P} 0$, and is similar to $\mathrm{P} 0$ at $\mathrm{S} 7-1$. For 
$\mathrm{P} 2$, the change characteristics of longitudinal flow velocity in the open channel are similar to that of P1. Since the height of $\mathrm{P} 2$ is significantly larger than that of $\mathrm{P} 1$, the change of flow velocity is not only greater than that of P1, but also the recovery distance in downstream region is significantly longer than that of P1. For the emergent vegetation (P3), the longitudinal velocity in the zone below the height of $0.3 \mathrm{z} / \mathrm{H}$ along the stream-wise is all increase to some extent compared with that of $\mathrm{P} 0$, especially that of at S4-1. Due to different types of vegetation with P1 and P2, the recovery distance of $\mathrm{P} 3$ is also significantly longer than that of $\mathrm{P} 1$ and $\mathrm{P} 2$, and not yet been fully approached to that of $\mathrm{P} 0$ at $\mathrm{S} 10-1$.

There are different vertical distributions of longitudinal flow velocity along the stream-wise in the different types of vegetated channel. For submerged vegetation, the flow velocity decreases obviously in the zone under the top of vegetation due to its strong flow resistance, while the flow velocity in the zone above the top of vegetation is increased obviously and basically complies with the logarithmic distribution. Affected by the exchange of water fluxes in different vertical zones of the downstream region, the flow is restructured and gradually restored. For emergent vegetation, the whole flow resistance is significantly higher than that of submerged vegetation due to its higher height. However, the emergent vegetation is generally composed of stem and foliage. Because the water blocking area of the stem is significantly smaller than that of the foliage, the flow velocity in the zone below the height of stem in the open channel will increase obviously, Meanwhile, the flow velocity will decrease significantly in zone of foliage, and the more closely to the vegetated region, the greater variation of flow velocity in the two vertical zones will be. The flow will undergo vigorous momentum mixing exchange in the two vertical zones, result in obvious secondary flow, and gradually return to the flow pattern of non-vegetation along the stream-wise in the downstream region.

(2) Flow velocity in transverse direction

In order to study the distribution of flow velocity in transverse direction of the partly vegetated channel, cross-section S1, S3, S4, S6, S8 and S10 are selected. Since the cross-sections and the arrangement of vegetation are strictly symmetrical along the midline of the flume, hence only half of the cross-sections of the open channel are measured. Five vertical measurement lines are arranged on each measured section, and the transverse distance of the measurement lines from the midline of the flume is $0 \mathrm{~cm}$, $4 \mathrm{~cm}, 8 \mathrm{~cm}, 15 \mathrm{~cm}, 21 \mathrm{~cm}$ (Figure 4). As shown in Figure 7, the transverse distribution of flow velocity of every cross-section in the different types of the partly vegetated channel is basically similar. Normally, the flow velocity in transverse direction decreases gradually as approaching from the midline to sidewall of flume, which mainly due to the relatively large flow resistance on the sidewall of the flume. However, for P3, due to its larger flow resistance, near the sidewall, the flow velocity in some vertical zones $(0-0.4 \mathrm{z} / \mathrm{H})$ is greater than that of in the middle of the flume. There is no vegetation in the downstream region, where the resistance of the sidewall is the main part of the whole flow resistance, since the flow velocity at different transverse positions is affected differently by the sidewall of the flume, which results in the 
significantly different recovery of flow velocity at different transverse positions. For example, when the velocity of vegetated flow is greater than that of non-vegetated flow, the distribution of velocity near the sidewall recovers more rapidly. In contrast, when the velocity of vegetated flow is smaller than that of non-vegetated flow, the distribution of velocity near the sidewall recovers more slowly.
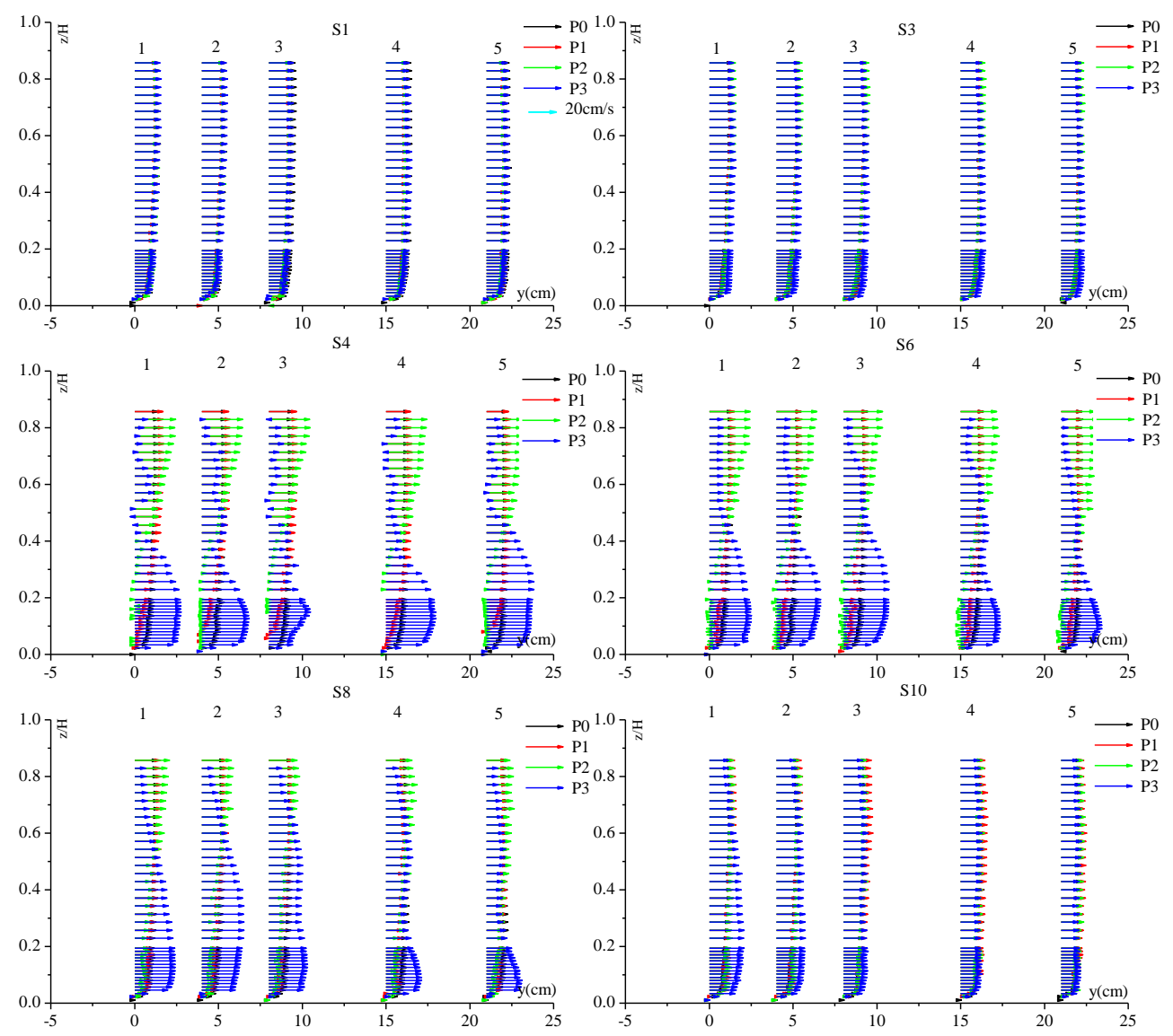

Figure 7. The distribution of flow velocity at different transverse positions in the vegetated open channel

(3) The regularity of influence

According to the experimental data of ten measured cross-sections, the regular influence on flow by different types of vegetation is analyzed. Considering that the regular influence of the flow characteristic at different vertical positions maybe not the same, three positions in the vertical direction are selected to be analyzed in this paper, which represent the bottom flow, the middle flow and the surface flow respectively. As shown in Figure 8, in terms of the range of influence, the influence on flow by different types of vegetation is greater in the downstream region than that in the upstream region. In terms of the degree of influence, all types of vegetation have great effects on the flow at the bottom position, followed by the surface position, and then the middle position. 
The relative flow velocity of different types of vegetation is ( $\mathrm{P}^{*}$-P0) /P0 (*represented the type of vegetation), the variation range of which near bottom, the surface and the middle is $-116 \%-161 \%,-85 \%-68 \%$ and $-73 \%-56 \%$, respectively. The middle zone is the main place where the flow from bottom and surface mix intensively. Therefore, the distribution of relative flow velocity in the middle zone is more uniform than that in the other two zones. For different types of vegetation, the submerged and emergent vegetation have completely opposite effects on the bottom flow and the surface flow. Relative to submerged vegetation, emergent vegetation has a greater influence on flow and longer influenced distance.
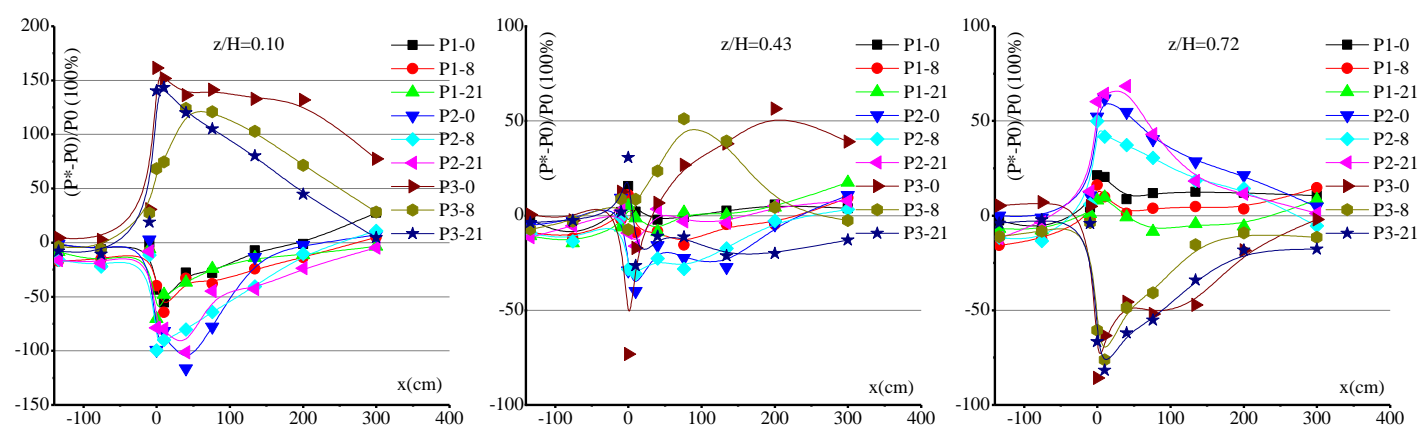

Figure 8. The regular influence on the flow in the different vegetated open channel

\section{Secondary flow}

The secondary flow is a relatively minor flow superimposed on the primary flow; however, it plays a crucial role in the sediment transport of the open channel. In this section, the secondary flow in the different types of the vegetated open channel is still investigated.

As can be seen from Figure 9, the secondary flow propagates from left sidewall to the centre of the flume at section S1 (left half of the flow) under different types of vegetation. Since the flume is strictly symmetrical with respect to the central line, the right half of the flow should propagate from right sidewall to the centre of the flume. The velocity of the secondary flow of section S1 in the vegetated open channel is larger than that of in non-vegetated open channel. Although there is a significant effect of vegetation on the secondary flow in section S1, the movement characteristic of the secondary flow does not change fundamentally.

In section S3, for the submerged vegetation (P1 and P2), because of its large flow resistance in the bottom and middle parts of the section (a place full of vegetation), the vertical distribution of secondary flow obviously propagates from the bottom to the free surface. For the emergent vegetation (P3), the vertical distribution of secondary flow obviously propagates from the free surface to the bottom for its large flow resistance in the middle and upper part of the section (a place full of foliage). However, in the bottom part of the section S3 (a place full of stem), the transverse flow of P3 is clearly shifted 
towards both sides of the plant stems, which is similar to the flow movement of the cylindrical spoiler (Figure 10), and the similar phenomenon we still can get from Graf and Yulistiyanto, 1998.

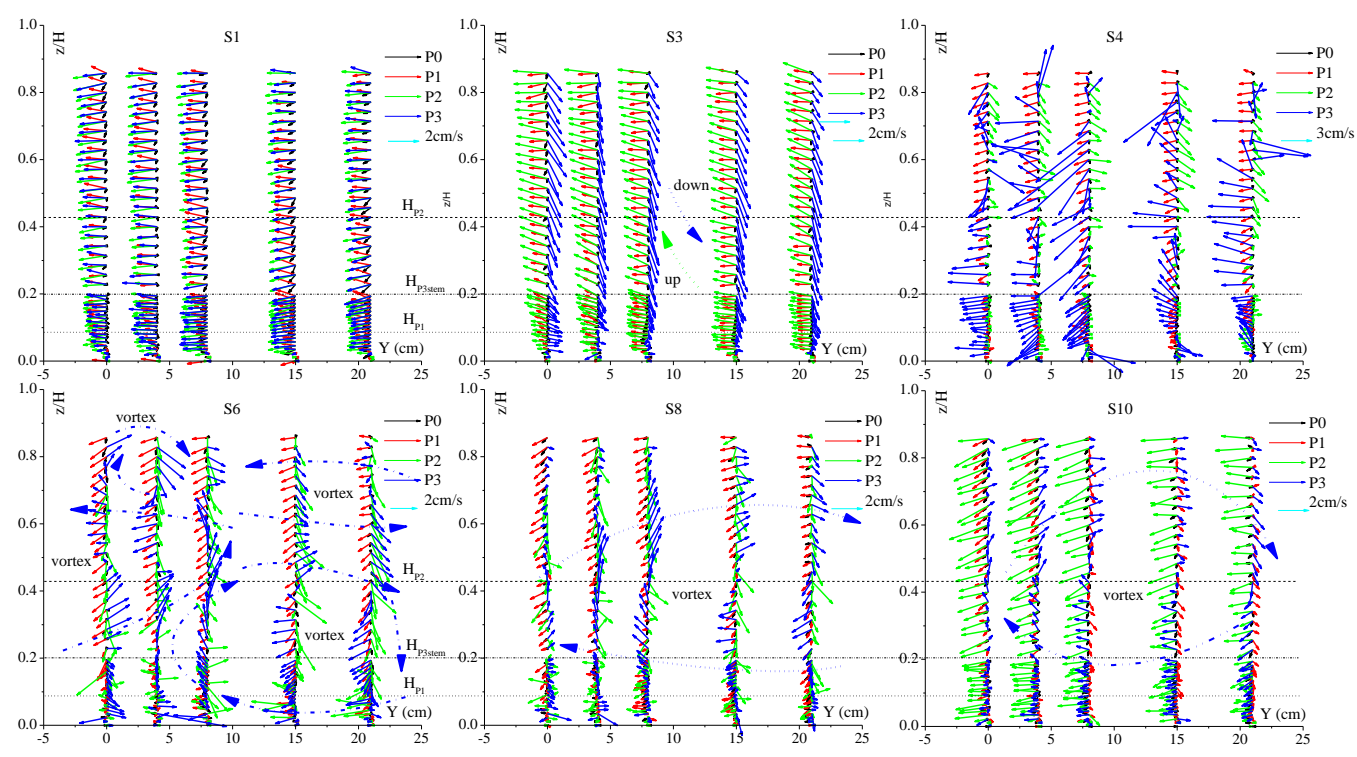

Figure 9. The vertical distribution of secondary flow in the vegetated open channel

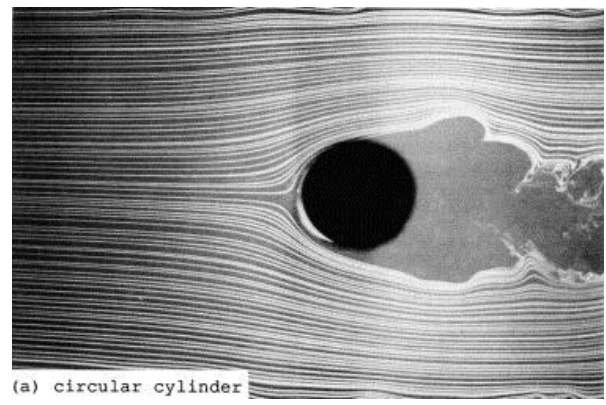

Figure 10. The flow pattern around circular cylinder at $R e=3 \times 10^{4}$, reprinted from Yoon \& Ettema (1993)

In section S4, where the vertical distribution secondary flow becomes more complicated due to the strong influence. For submerged vegetation (P2), the velocity of secondary flow in the zone under the height of $0.8 \mathrm{HP} 2$ is small, whereas the velocity in the zone above the height of 0.8 HP2 is obviously increased. At the same time, the transverse direction of secondary flow in these two zones is not strictly consistent, which may be the result of the exchange of flow momentum and the shear layer near the canopy of submerged vegetation (Figure 11). For emergent vegetation (P2), its flow resistance is significantly different in the zones of stems and foliage. In the zone of foliage, the secondary flow is more significant mutations scattered and occurred in some 
local positions. However, in the zone of stems, the secondary flow is less influenced and has a regular flow pattern. Furthermore, the secondary flow of P3 propagates from left sidewall to centre of the flume at the majority positions in section $\mathrm{S} 4$, whose direction is opposite to that in the section S3. The reason for this phenomenon might be related to the vortex flow generated in the vegetation area (see Figure 12b).

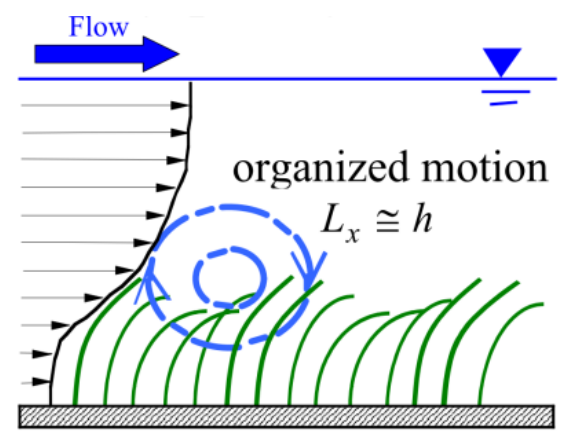

Figure 11. The flow patterns in dense flexible vegetation (Okamoto \& Nezu 2010)

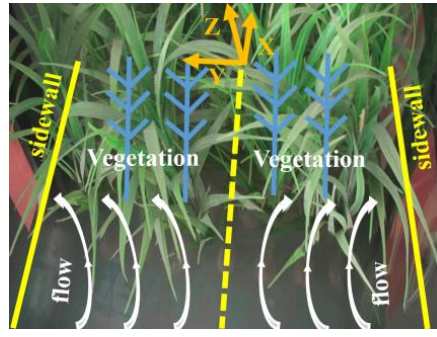

(a) $\mathrm{S} 3$

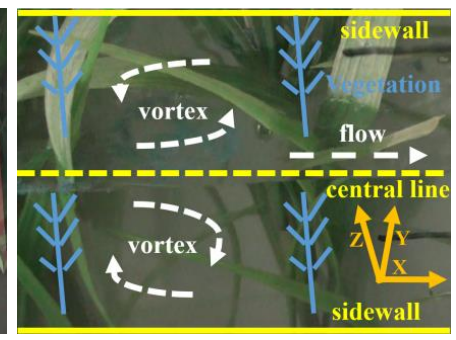

(b) S4

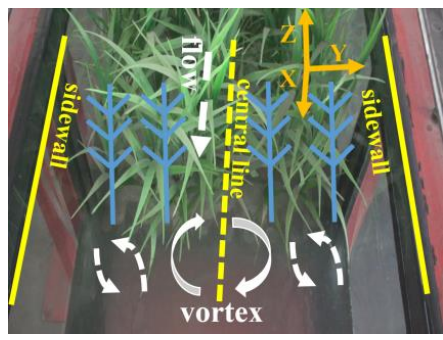

(c) S6

Figure 12. The characteristic of secondary flow of different region under the condition of P3

In the downstream region (S5-S10), due to the influence of vegetation, there is a significant momentum exchange among the water masses in different vertical positions and significant vortex flow is generated. In order to further analyze the characteristics of vortex flow in the downstream region, this paper selects three sections (S6, S8 and S10) and calculates the measured data by Equation (1), finally obtains the vertical distribution of vortex flow in the longitudinal direction. For the purpose of making the result clearer and more intuitive, more significant distribution of vorticity is taken as an example (vegetation P3). As shown in Figure 13, six vortices are generated in the flow field of section S6, which indicates that there are some strong vertical exchanges of flow momentum. Obviously, in the case of sediment, this condition of flow is conducive to sediment diffusion in the three-dimensional direction and makes sediment concentration consistent in the whole section. With the mix of flow in the downstream region, the energy of flow gradually decreases, and the flow converges and merges into two vortices in section S8. Finally, there is only one large vortex in section S10 and the vorticity intensity is further weakened. 


$$
\Omega_{x}=\frac{\partial w}{\partial y}-\frac{\partial v}{\partial z}
$$

As previously analyzed, due to the influence of vegetation, the flow in the downstream region generates many significant vortices. Compared to the submerged vegetation, emergent vegetation has more obvious influence on the flow in the downstream region. Therefore, in terms of ecological rivers, the presence of intense vortex flow in downstream of emergent vegetation region will make the substances in rivers fully diffused, which is obviously conducive to the survival and propagation of aquatic organisms.

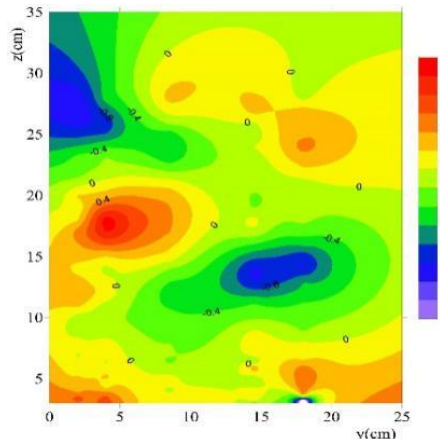

(a) S6

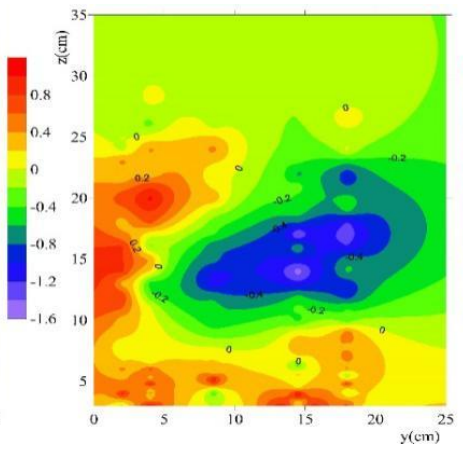

(b) $\mathrm{S} 8$

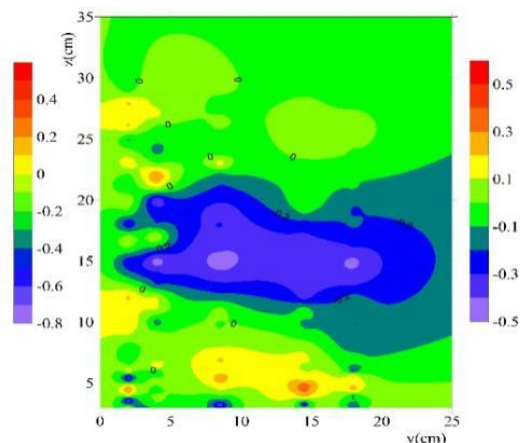

(c) S10

Figure 13. The vertical distribution of vortex flow in longitudinal direction under the condition of $P 3$

\section{Sediment transport}

According to the analysis of the data measured by rivers and laboratory experiments, $\mathrm{Hu}$ and Hui (1995) considered that the vertical distribution of sediment concentration in open channel usually had three types. Type I distribution is that the sediment concentration increases gradually from the free surface to the riverbed, and when it reaches a maximum value at a certain vertical position, then it will decrease. Type II distribution is that the sediment concentration monotonically increases from the free surface to the riverbed, with the maximum concentration near the riverbed. Type III distribution is that the sediment concentration increases gradually from the free surface to the riverbed, with the maximum concentration still near the riverbed, and yet the sediment gradient will vary significantly at one vertical position. Ni et al. (1991) considered that the vertical pulsating flow velocity was the fundamental reason for the existence of suspended sediment in a turbulent flow. If the sediment concentration is not very large, there will be more opportunity for Type I distribution when the bed is smooth with the uniform particle size of sediment. On the contrary, there will be more opportunity for Type II distribution. 
Hu and Hui (1995) considered that the appearance of Type I distribution could not be explained by only the vertical pulsatile flow velocity distribution. Whether Type I distribution occures in flow with a higher concentration depends on the ratio of particle size to flow depth, and if the ratio exceeds the critical value the flow may appear Type I distribution. The formation condition of Type II distribution is that the suspension index is large and the gravity plays a dominant role, while the formation condition of Type III distribution is that the suspension index is small and the turbulent diffusion plays a dominant role. In order to study the vertical distribution of suspended sediment transport in the different vegetated open channel, laboratory experiments are carried out. The experimental conditions are shown in Table 1. The median diameter of experimental sediment ranges from $0.16 \mathrm{~mm}$ to $0.18 \mathrm{~mm}$, and the sedimentation velocity is calculated by Zhang Ruijin formula (Qian and Wan, 1986). Experimental samples are obtained by sediment sampler (Figure 1). The Samples are taken twice from every measured position by $1000 \mathrm{ml}$ graduated cylinders, filtered and dried, and then are weighed by an electronic balance. Finally, the sediment concentration of the sample is calculated and the average of two results in the same position is taken as the sediment concentration of the measured position.

\section{Suspended sediment concentration}

According to the experimental data in three vertical lines of S1-1, S4-1 and S8-1, the vertical distribution of the suspended sediment concentration in the different types of the vegetated open channel is analyzed. As shown in Figure 14, the vertical distribution of the suspended sediment concentration under different types of vegetation in the upstream region (at S1-1) is basically satisfies Type I and Type II distribution, which is similar to that of in the middle reaches of Changjiang River (Cross-section JM01, Time 24 June 2016, Discharge $17900 \mathrm{~m}^{3} / \mathrm{s}$, Water depth $14.5 \mathrm{~m}$, Depth-averaged velocity $1.47 \mathrm{~m} / \mathrm{s})$. In the vegetated region $\left(\mathrm{H}_{2}-\mathrm{H}_{3}\right)$, due to the influence by vegetation, the vertical distribution of the suspended sediment concentration changes greatly relative to that in the upstream region. The value of vertical sediment concentration under conditions of P2 and P3 decreases significantly and vertical change characteristic satisfies Type III distribution. In downstream region (at S8-1), the vertical concentration of suspended sediment under different types of vegetation is distributed more evenly.

The vertical distribution of suspended sediment gradient is an important factor that represents the variation of suspended sediment transport. Since the distribution of suspended sediment concentration is more scattered than that of flow velocity, the vertical distribution of suspended sediment concentration will not be simulated in this paper, instead of being obtained by differentiating the experimental data (Figure 15). As shown in Figure 15, the sediment gradient in the zone under the height of $0.2 \mathrm{z} / \mathrm{H}$ is relatively large under the condition of vegetation, while the value of suspended sediment gradient in the zone above the height of $0.2 \mathrm{z} / \mathrm{H}$ gradually decreases and is basically negative. For P1, the vertical distribution of suspended sediment gradient in 
the vegetated open channel is basically the same as that of $\mathrm{P} 0$, which indicates that the vegetation (P1) has a relatively slight influence on the suspended sediment transport.

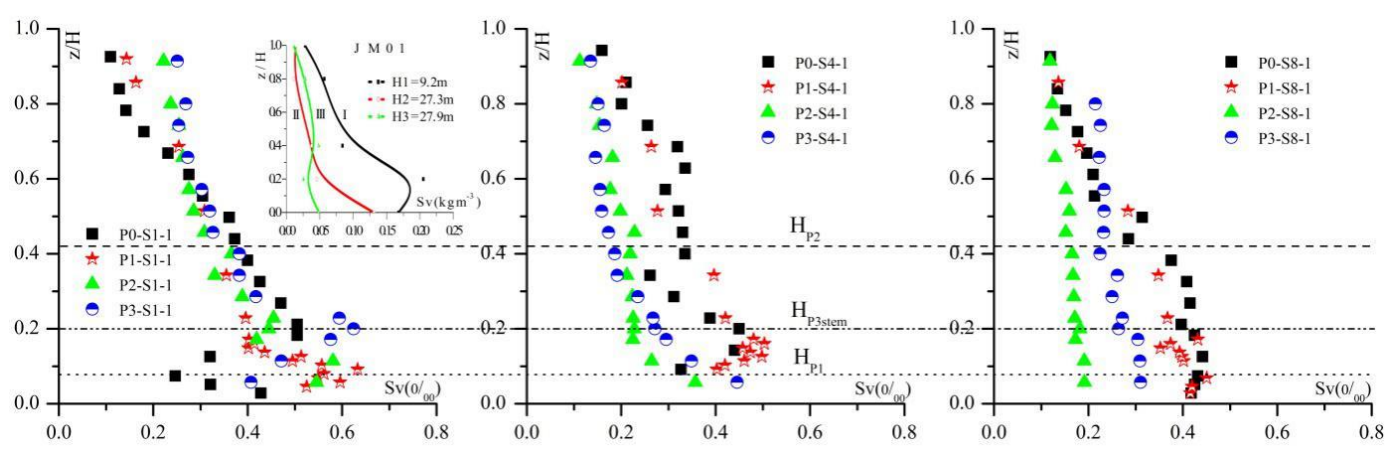

Figure 14. The vertical distribution of the suspended sediment concentration in the different types of the vegetated open channel

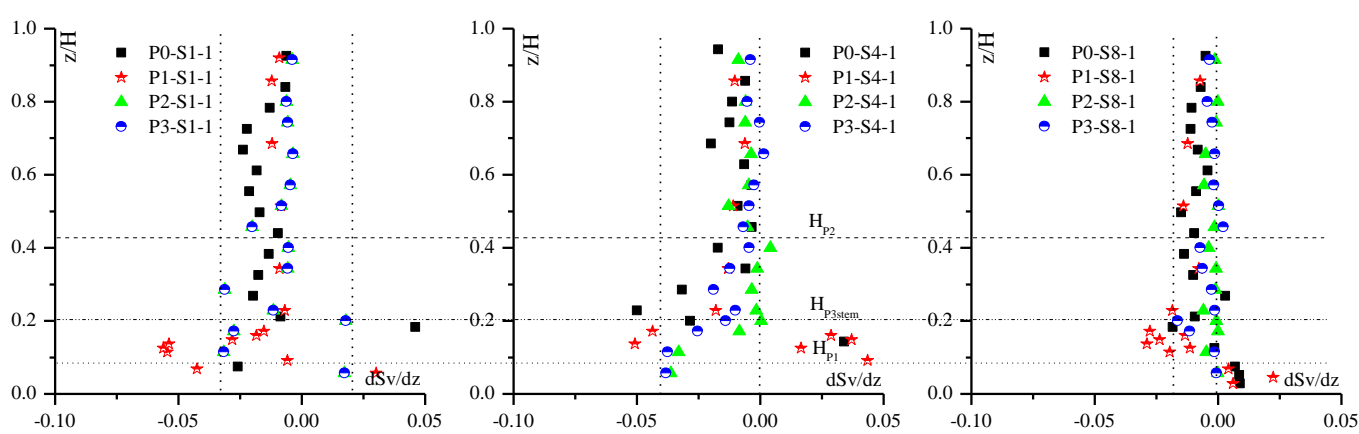

Figure 15. The vertical distribution of the suspended sediment gradient in the different types of the vegetated open channel

However, for P2 and P3, the vertical distribution of suspended sediment gradient gradually decreases along the stream-wise, that is to say, the vertical distribution of suspended sediment concentration tends to be consistent at S4-1 and S8-1. There are two possible reasons to explain the significant change of characteristics under conditions of P2 and P3. First, influenced by the water blocking of vegetation (P2 and P3), the large particles of suspended sediment are largely deposited in the upstream and vegetated region and the particle size of suspended sediment along the stream-wise becomes smaller and smaller, which will be conducive to the sediment suspension in the flow. Second, for P2 and P3, the turbulent diffusion of flow at S4-1 and S8-1 is significantly stronger than that of $\mathrm{P} 0$, which will be beneficial to the diffusion of suspended sediment in the flow. 


\section{Particle size of sediment}

Malvern Mastersizer 2000 particle size analyzer is used to analyze the particle size of suspended sediment, and then the vertical distribution about different particle size of suspended sediment (less than $0.1 \mathrm{~mm}$ fine sediment particles and more than $0.1 \mathrm{~mm}$ coarse sediment particles) is analyzed. As shown in Figure 16, the size of suspended sediment median particles gradually diminishes from bottom to free surface. However, for P2 and P3, the vertical distribution about the size of suspended sediment median particles at S3-1 does not have significant changes, which should be related to their greater turbulence intensity of the flow in the downstream region.
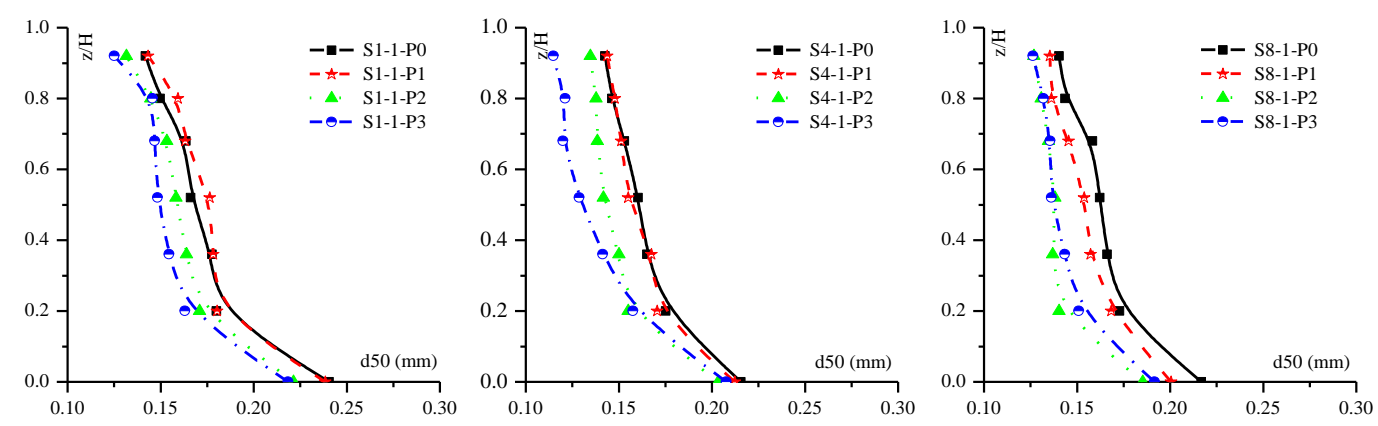

Figure 16. The vertical distribution of suspended sediment median particles size $\left(d_{50}\right)$

As can be seen from Figure 17, the concentration of fine sediment particles does not change obviously along with the water depth. Therefore, the vertical distribution of fine sediment particles is not related to the type of vegetation and the location of the vegetated open channel.
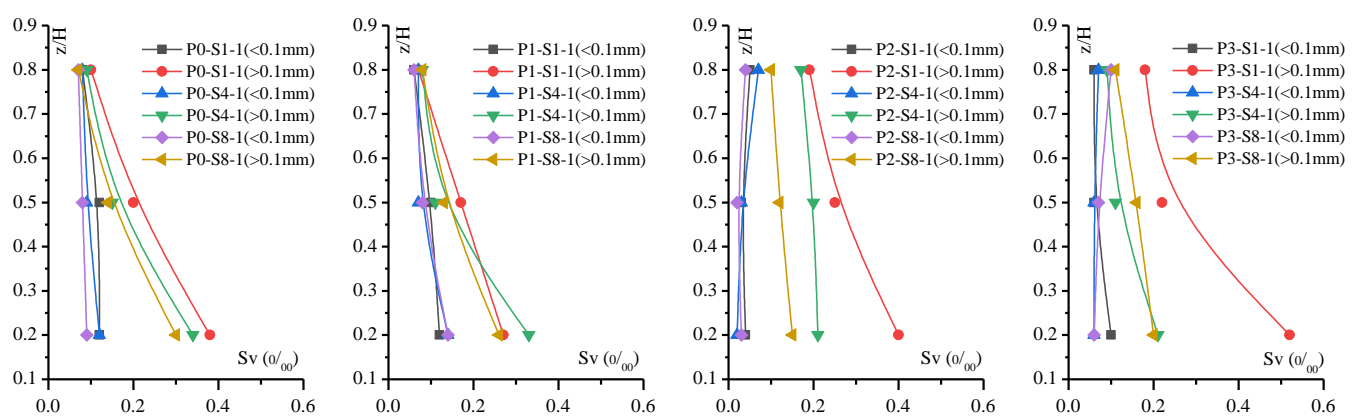

Figure 17. The vertical distribution about concentration of different suspended sediment particles size

On the contrary, for coarse sediment particles, the concentration decreases obviously along with the water depth. Taking P2 and P3 for example, the coarse sediment at S1-1 rapidly decreases with the increase of water depth, yet slowly decreases at S2-1 and S31. There are two possible reasons to explain the different changes of coarse sediment. 
First, coarse sediment is largely deposited in the upstream and vegetated region due to the water blocking the impact of the vegetation. Second, the turbulence of flow in the two regions is strong and the vertical dispersion of suspended sediment is very significant. Experiments show that vegetation has a weak filtration effect on fine sediment in the open channel, but has a strong filtration effect on the coarse sediment. The proportion of fine sediment in a total load along the stream-wise gradually increases, in contrast, the proportion of coarse sediment in a total load along the streamwise decreases gradually. As a result, the suspended sediment along the stream-wise in the vegetated open channel is gradually refined.

\section{Discussion}

\section{Flow and sediment diffusion coefficient}

Due to the turbulent characteristics of the flow, sediment transport is actually bidirectional in the process of turbulent diffusion. Under certain conditions, the total amount of sediment entering the lower concentration zone is equal to the total amount of sediment entering the higher concentration zone, at which time the distribution of sediment concentration reaches equilibrium. The reason for this balance is that the upward turbulence force of sediment is equal to the downward gravity $(\mathrm{Ni}, 1991)$. The sediment diffusion coefficient and flow diffusion coefficient are important indicators of flow and sediment diffusion intensity. This paper attempts to study the mechanism of interaction between flow and sediment in the partly vegetated open channel through the analysis of flow diffusion and sediment diffusion coefficient. According to the momentum transfer theory of turbulence (Ni et al., 1991) and diffusion equation of twodimensional constant uniform flow under equilibrium conditions, formulas for calculating momentum diffusion coefficient $\left(\varepsilon_{m}\right)$ and sediment diffusion coefficient can

be derived $\left(\varepsilon_{s}\right)$.

As shown in Figure 18, the vertical distribution of flow momentum diffusion coefficient increases firstly and then decreases in different types of the vegetated open channel. At S1-1, the greater flow resistance of vegetation is, the smaller value of flow momentum diffusion coefficient along the water depth is. For P2 and P3, the value of flow momentum diffusion coefficient is obviously smaller than that of P0 and P1. Table 2 shows the correlations of flow momentum diffusion coefficient under different types of vegetation. The correlations between P0 and P1, P2, P3 are 0.81, 0.58 and 0.90, respectively. Obviously, the correlation between $\mathrm{P} 0$ and $\mathrm{P} 2$ is very low, which indicates that vegetation $\mathrm{P} 2$ has the greatest influence on the flow momentum diffusion coefficient in the upstream region. In the vegetated region $\left(\mathrm{H}_{2}-\mathrm{H}_{3}\right)$, due to the effect of vegetation $\mathrm{P} 2$ and $\mathrm{P} 3$, the values of flow momentum diffusion coefficient in some vertical zones are significantly larger than those in upstream. The correlation between P0 and P3 also significantly reduces compared with those in upstream (Table 2). In the 
downstream region (at S8-1), for P2 and P3, the vertical distribution of flow momentum diffusion coefficient is basically similar to that of $\mathrm{P} 0$, however, the maximum value along the water depth is obviously larger than that of $\mathrm{P} 0$, and the correlation between $\mathrm{P} 0$ and P2 also increases significantly relative to that in the vegetated region, yet the correlation between P0 and P3 is still small. The above change indicates that the influence on flow in the submerged vegetated open channel is mainly in the upstream and vegetated region, while that in the submerged vegetated open channel is mainly in the vegetated and downstream region.

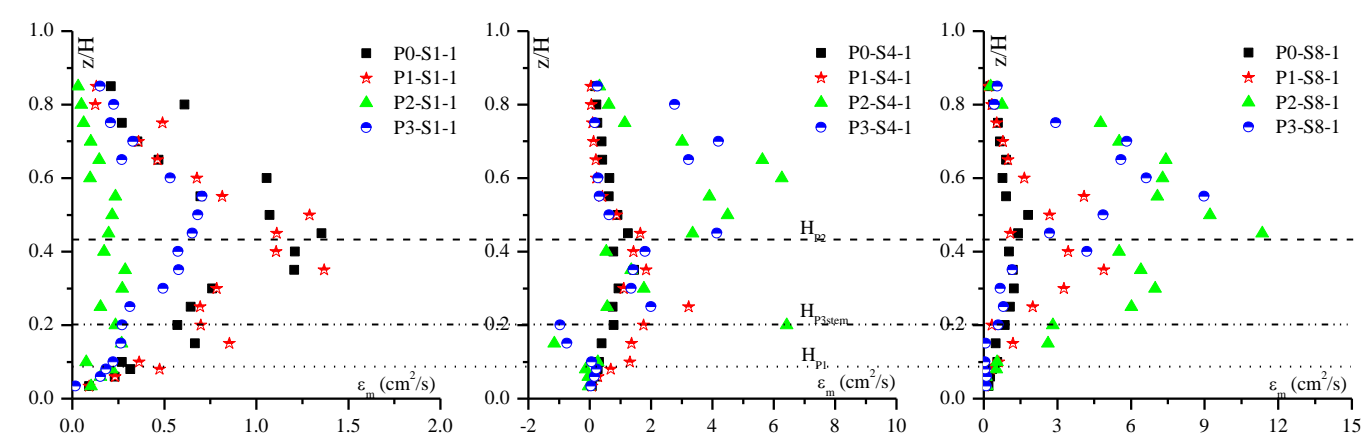

Figure 18. The vertical distribution of momentum diffusion coefficient along the stream-wise

Table 2. The Correlation of flow and sediment diffusion under different types of vegetation

\begin{tabular}{cc|c|c|c|c|c|c|c|c|c|c}
\hline \multirow{2}{*}{ Position factor } & \multicolumn{3}{|c|}{$\begin{array}{c}\text { Correlation of flow diffusion } \\
\text { coefficient }\end{array}$} & \multicolumn{3}{c|}{$\begin{array}{c}\text { Correlation of Sediment } \\
\text { diffusion coefficient }\end{array}$} & \multicolumn{3}{c}{$\begin{array}{c}\text { Correlation of flow and sediment } \\
\text { diffusion in every case }\end{array}$} \\
\cline { 3 - 14 } & & P0-P1 & P0-P2 & P0-P3 & P0-P1 & P0-P2 & P0-P3 & P0-P0 & P1-P1 & P2-P2 & P3-P3 \\
\hline \multirow{2}{*}{ S1-1 } & Corr & 0.81 & 0.58 & 0.90 & 0.64 & 0.22 & -0.19 & 0.55 & 0.76 & -0.13 & 0.39 \\
\cline { 2 - 14 } & Sig & $2.6 \mathrm{E}-05$ & $9.7 \mathrm{E}-03$ & $1.5 \mathrm{E}-07$ & $7.1 \mathrm{E}-03$ & $4.1 \mathrm{E}-01$ & $4.7 \mathrm{E}-01$ & $2.6 \mathrm{E}-02$ & $5.8 \mathrm{E}-04$ & $6.3 \mathrm{E}-01$ & $1.3 \mathrm{E}-01$ \\
\hline \multirow{2}{*}{ S4-1 } & Corr & 0.75 & 0.60 & 0.40 & 0.89 & 0.82 & -0.41 & 0.84 & 0.56 & 0.38 & 0.24 \\
\hline \multirow{2}{*}{ S8-1 } & Sig & $1.9 \mathrm{E}-04$ & $6.6 \mathrm{E}-03$ & $8.7 \mathrm{E}-02$ & $3.2 \mathrm{E}-06$ & $8.5 \mathrm{E}-05$ & $1.1 \mathrm{E}-01$ & $5.0 \mathrm{E}-05$ & $2.3 \mathrm{E}-02$ & $1.4 \mathrm{E}-01$ & $3.7 \mathrm{E}-01$ \\
\hline & Corr & 0.86 & 0.88 & 0.58 & 0.88 & -0.26 & -0.54 & 0.63 & 0.58 & 0.15 & 0.45 \\
\hline
\end{tabular}

As shown in Figure 19 and Table 2, the vertical distribution of sediment diffusion coefficients is obviously more complex than that of the flow momentum diffusion coefficient in different types of the vegetated open channel. For P1, the vertical distribution of sediment diffusion coefficients along the stream-wise have a high correlation with that of P0. For P2, the vertical distribution of sediment diffusion coefficients has a high correlation with that of $\mathrm{P} 0$ in the vegetated region $\left(\mathrm{H}_{2}-\mathrm{H}_{3}\right)$. However, the correlation between P2 and P0 in the upstream and downstream regions are significantly low. For P3, the vertical distribution of sediment diffusion coefficients in the whole open channel has a low correlation with that of $\mathrm{P} 0$, and all of the correlations are negatively correlated. 


\section{Mechanism of interaction between flow and sediment}

The reason why suspended sediment can move forward with the flow is mainly due to the contrast between the turbulent diffusion of flow and the gravity of sediment $(\mathrm{Hu}$ and Hui, 1995). The process of suspended sediment transport is the process of the interaction between turbulent diffusion and sediment gravity. The experimental analysis also shows that there is a medium to the high degree of correlation between the momentum and sediment diffusion coefficients in the non-vegetated open channel (Table 2). The mechanism of the interaction between flow and sediment in open vegetated channel is very complex. When the morphology of vegetation is relatively small, it has little effect on the process of flow and sediment transport in the open channel.

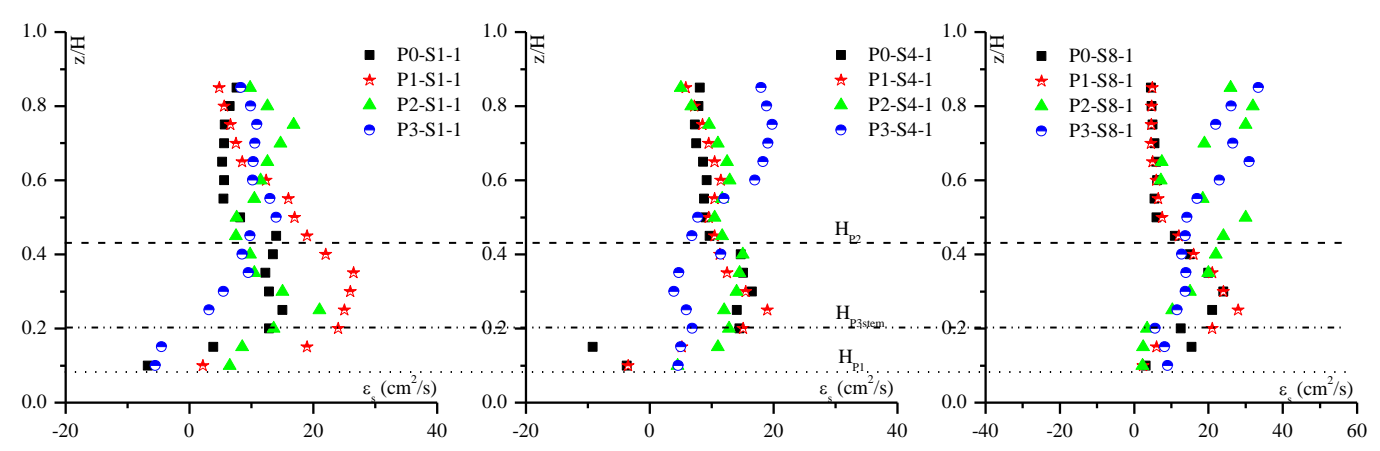

Figure 19. The vertical distribution of sediment diffusion coefficient along the stream-wise

However, when the morphology of vegetation is relatively large, its influence on the process of flow and sediment transport in the open channel is greater. At this time, the correlation between the flow and sediment transport is significantly reduced and even negative correlation occurs. With the increased volume of the vegetation, the area of water blocking in the cross-section gradually increases and the turbulence intensity of flow also significantly increases in the open channel, which leads to the obvious threedimensional vortex flow in some zones. The existence of three-dimensional vortex flow is conducive to the diffusion of sediment in different directions. Since the calculated flow diffusion coefficient in this paper does not consider the influence of transverse flow in the open channel, yet the distribution of sediment concentration in the vegetated open channel is caused by the three-dimensional movement of the flow, so the correlations between the flow momentum diffusion coefficient and the sediment diffusion coefficient in some areas are low or not relevant. All these analyses show that the movement of transverse flow in the partly vegetated open channel has a crucial impact on suspended sediment transport. 


\section{Conclusions}

Experiments are conducted on characteristics of the flow in the partly vegetated channel. For submerged vegetation, the flow velocity decreases obviously in the zone under the top of vegetation due to its strong flow resistance, while the flow velocity in the zone above the top of vegetation increases obviously and basically complies with the logarithmic distribution. The place slightly below the top of submerged vegetation can be regarded as the "riverbed", and is called "vegetation-equivalent riverbed". For emergent vegetation, the place where the vertical distribution of the longitudinal flow velocity fluctuates strongly is the transition zone between the stem and foliage, therefore, the upper edge of the transition zone can be regarded as the "equivalent riverbed" for the emergent vegetated channel.

The regular influence on flow in the partly vegetated open channel is analyzed. In terms of the range of influence, the influence on the flow of different types of vegetation is greater in the downstream region than that in the upstream region. In terms of the degree of influence, all types of vegetation have a great effect on the flow at the bottom position, followed by the surface position, and then the middle position. For different types of vegetation, the submerged and emergent vegetation has completely opposite effect on the bottom flow and the surface flow. Relative to submerged vegetation, emergent vegetation has a greater influence on flow and longer influenced distance.

Due to the influence of vegetation, the flow in the downstream region generates many significant vortices. Compared with the submerged vegetation, emergent vegetation has a more significant influence on the flow in the downstream region. Therefore, in terms of ecological rivers, the presence of intense vortex flow in the downstream region will make the substances in rivers fully diffused, which is obviously conducive to the survival and propagation of aquatic organisms.

The vertical distribution of suspended sediment in the partly vegetated channel becomes more and more uniform along the stream-wise. Besides, the vertical variation of the suspended sediment gradient decreases gradually along the stream-wise. Vegetation has a weak filtration effect on fine sediment in the open channel but has a strong filtration effect on the coarse sediment. The proportion of fine sediment in a total load along the stream-wise gradually increases; in contrast, the proportion of coarse sediment in a total load along the stream-wise decreases gradually. As a result, the suspended sediment along the stream-wise in the partly vegetated open channel is gradually refined.

The mechanism of the interaction between flow and sediment in the partly vegetated channel is very complex. When the morphology of vegetation is relatively small, it has little effect on the process of flow and sediment transport in the open channel. However, when the morphology of vegetation is relatively large, its influence on the process of flow and sediment transport in the open channel is greater. At this time, the correlation between the flow and sediment movement is significantly reduced and even negative correlation occurs. These show that the movement of transverse flow in the vegetated open channel has a crucial impact on suspended sediment transport. 
In this paper, the vegetation is made by flexible plastics. Although the plastic vegetation has some flexibility, it is still different from natural aquatic vegetation in stiffness. To further improve the research, it is necessary to use real aquatic vegetation to carry out future experiments. For future experiments, the aquatic vegetation can be planted in the flume in advance. The effects on the flow and sediment transport can be studied separately according to the different growth stages of aquatic vegetation.

Acknowledgements. The research is supported by National Key Research and Development Program of China (under Grant No. 2016YFC0402310, No. 2016YFC0402105), National Natural Science Foundation of China (under Grant No. 51679011, No. 51309022, No. 51579172).

\section{REFERENCES}

[1] Carollo, F. G., Ferro, V., Termini, D. (2002): Flow velocity measurements in vegetated channels. - Journal of Hydraulic Engineering 128: 664-673.

[2] Elliott, A. (2000): Settling of fine sediment in a channel with emergent vegetation. Journal of Hydraulic Engineering 126: 570-577.

[3] Graf, W. H., Yulistiyanto, B. (1998): Experiments on flow around a cylinder; the velocity and vorticity fields. - Journal of Hydraulic Research 34: 637-653.

[4] Hu, C., Hui, Y. (1995): Mechanisms and statistical laws of flow and sediment movement in open channel. - Science Press, Beijing, China.

[5] Ikeda, S., Kanazawa, M. (1996): Three-Dimensional Organized Vortices above Flexible Water Plants. - Journal of Hydraulic Engineering 122: 634-640.

[6] Jordanova, A. A., James, C. S. (2003): Experimental Study of Bed Load Transport through Emergent Vegetation. - Journal of Hydraulic Engineering 129: 474-478.

[7] Li, R. M., Shen, H. W. (1973): Effect of Tall Vegetations on Flow and Sediment. Journal of Hydraulic Division 99: 793-814.

[8] Li, Y., Du, Wei., Yu, Z. (2015): Impact of flexible emergent vegetation on the flow turbulence and kinetic energy characteristics in a flume experiment. - Journal of Hydroenvironment Research 9: 354-367.

[9] Lu, S. (2008): Experimental Study on Suspended Sediment Distribution in Flow with Rigid Vegetation. - Hohai University, Nanjing, China.

[10] Nezu, I., Onitsuka, K. (2001): Turbulent structures in partly vegetated open-channel flows with LDA and PIV measurements. - Journal of Hydraulic Research 39: 629-642.

[11] Ni, J., Wang, G., Zhang, H. (1991): The theory of sediment and flow transport and the newest applications. - Science Press, Beijing, China.

[12] Okamoto, T., Nezu, I. (2010): Flow resistance law in open-channel flows with rigid and flexible vegetation. - Bundesanstalt für Wasserbau, Karlsruhe, Germany.

[13] Qian, N., Wan, Z. (1986): Sediment movement mechanics. - Science Press, Beijing, China.

[14] Qu, G. (2014): Experimental Study on Water and Sediment Transport in Open Channel Flow with Vegetation. - Wuhan University, Wuhan, China. 
[15] Shi, B., Cao, S. (2000): The Control Sand Dynamics Using Vegetation. - Ocean University of China Press, Qingdao, China.

[16] Stephan, U., Gutknecht, D. (2002): Hydraulic resistance of submerged flexible vegetation. - Journal of Hydrology 269: 27-43.

[17] Su, X., Li, C. (2002): Large eddy simulation of free surface turbulent flow in partly vegetated open channels. - International Journal for Numerical Methods in Fluids 39: 919-937.

[18] Sun, G., Wei, G. (2005): The study on model sediment experiment in Changjiang River flood control model. - Changjiang River Scientific Research Institute, Wuhan, China.

[19] Tang, H., Yan, J., Lu, S. (2007): Advances in research on flows with vegetation in river management. - Advances in Water Science 18: 785-792.

[20] Wang, X., Shao, X., Li, D. (2002): The Basis of River Dynamics. - China Water \& Power Press, Beijing, China.

[21] Wilson, C., Stoesser, T., Pinzen, B. (2003): Open channel flow through different forms of submerged flexible vegetation. - Journal of Hydraulic Engineering 129: 847-853.

[22] Yang, S., Lee, J. (2007): Reynolds shear stress distributions in a gradually varied flow in a roughened channel. - Journal of Hydraulic Research 45: 462-471.

[23] Yoon, B., Ettema, R. (1993): Droplet trajectories and icing-collision efficiencies for cylinders determined using LDV. - Cold Regions Science and Technology 21: 381-397.

\section{GLOSSARY}

The following symbols are used in this paper:

$A_{v e}$ :Cumulative area of the whole plant $\left(\mathrm{cm}^{2}\right)$

$d_{50}$ :Median diameter of experimental sand $(\mathrm{mm})$

Fr:Froude number

$g$ :Gravitational constant $\left(\mathrm{m} / \mathrm{s}^{2}\right)$

$H$ :Water depth $(\mathrm{cm})$

$H_{0}$ :Water level control at the outlet of the flume $(\mathrm{cm})$

$H_{P I}:$ Height of vegetation $\mathrm{P} 1(\mathrm{~cm})$

$H_{P 2}$ :Height of vegetation $\mathrm{P} 2(\mathrm{~cm})$

$H_{P 3}:$ Height of vegetation $\mathrm{P} 3(\mathrm{~cm})$

$H_{P 3 s t e m}:$ Height of stem of vegetation P3 $(\mathrm{cm})$

$h_{v e}$ :Different height of vegetation $(\mathrm{cm})$

$J$ :Water surface slope

$Q$ :Flow discharge $(\mathrm{L} / \mathrm{s})$

Re:Reynolds number (-)

Sa:Amount of sediment at inlet of the rectangle flume $(\mathrm{g} / \mathrm{s})$

$\mathrm{S}_{\mathrm{L}}$ :Flume slope

$\mathrm{S}_{\mathrm{v}}:$ Sediment volume concentration $(1 / 1000)$

$u$ :Flow velocity of point $\mathrm{z}(\mathrm{cm} / \mathrm{s})$ 
$u_{a}:$ Time-averaged stream-wise velocity $(\mathrm{cm} / \mathrm{s})$

$u_{\max }:$ Maximum flow velocity in the depth direction $(\mathrm{cm} / \mathrm{s})$

$\mathrm{u}_{\mathrm{rms}}:$ Turbulence intensity $(\mathrm{cm} / \mathrm{s})$

$u_{\text {: }}$ : Shear velocity $(\mathrm{cm} / \mathrm{s})$

$u^{J}:$ Mean velocity components in longitudinal direction $(\mathrm{cm} / \mathrm{s})$

$v^{\prime}:$ Mean velocity components in transverse directions $(\mathrm{cm} / \mathrm{s})$

$w^{\prime}:$ Mean velocity components in vertical directions $(\mathrm{cm} / \mathrm{s})$

$v^{\prime} w^{\prime}$ : Mean velocity components in longitudinal, transverse and vertical directions, respectively $(\mathrm{cm} / \mathrm{s})$

$-u^{s} v^{\prime}$ : Reynolds stress in vertical direction (z) on plane perpendicular to stream-wise direction $\left(\mathrm{cm}^{2} / \mathrm{s}^{2}\right)$

$\mathrm{X}$ : Longitudinal direction

$\mathrm{x}$ : Distance to the vegetation region in longitudinal direction $(\mathrm{cm})$

Y: Transverse direction

$\mathrm{y}$ : Distance to the left sidewall of the flume $(\mathrm{cm})$

$\mathrm{Z}$ : Vertical direction

$\mathrm{z}$ : Distance to the riverbed $(\mathrm{cm})$

\section{Greek symbols}

$\varepsilon_{m}:$ Momentum diffusion coefficient

$\omega$ : Settling velocity of sediment $(\mathrm{cm} / \mathrm{s})$

$\kappa:$ Von Karman's turbulence coefficient

$\rho:$ Fluid density

$\varepsilon_{\varepsilon}:$ Sediment diffusion coefficient

$\Omega_{\mathrm{x}}$ : Vortex rotation in longitudinal direction. 